\title{
Karyotype analysis and genetic variation of a mutant in Siraitia grosvenorii
}

\author{
Wei Fu $\cdot$ Xiaojun Ma $\cdot$ Qi Tang $\cdot$ Changming Mo
}

Received: 14 January 2011/Accepted: 12 May 2011/Published online: 21 May 2011

(C) The Author(s) 2011. This article is published with open access at Springerlink.com

\begin{abstract}
This study analyzed the differences in karyotype and genetic variation between a mutant and wild-type $\mathrm{Si}$ raitia grosvenorii. Genetic variation included changes in genome and gene expression by SRAP molecular markers. Results showed that wild-type $S$. grosvenorii was diploid, with a chromosome number of $2 n=2 x=28$, whereas the mutant was tetraploid with a chromosome number of $2 n=4 x=56.4573$ DNA bands were obtained using 189 different primer combinations, 577 of which were polymorphic, averaging 3.1 bands for each primer pair, while 1998 pairs were identical. There were no apparent differences on bands amplified by most primer pairs. After comparing the diploid and tetraploid strains, the data generally indicated that the polymorphism would be quite low. 2917 cDNA bands were generated using 133 primer combinations, and stable and clearly differential fragments were sorted out, cloned and sequenced. Ninety-two differentially expressed fragments were successfully sequenced. Sequence analysis showed that most fragments had significant homologous nucleotide sequences with resistant to stress and photosynthesis genes, including ribulose-1,5bisphosphate carboxylase/oxygenase, phosphoenolpyruvate carboxykinase, pyruvate kinase, peroxisomal membrane
\end{abstract}

Electronic supplementary material The online version of this article (doi:10.1007/s11033-011-0855-6) contains supplementary material, which is available to authorized users.

\footnotetext{
W. Fu $\cdot$ X. Ma $(\bowtie)$

Institute of Medicinal Plant Development, China Academy Medicinal Science, Chinese Peking Union Medical College, 100193 Beijing, People's Republic of China

e-mail: xjma@public.bta.net.cn

Q. Tang · C. Mo

Guangxi Botanical Garden of Medicinal Plant, 530023 Nanning,

People's Republic of China
}

transporter, NBS-LRR type resistance protein, protein phosphatase and others. The results revealed that the tetraploid strain has more resistant and photosynthesis ability than its diploid relatives, which providing reference information and resources for molecular breeding and seedless Luohanguo.

Keywords Gene expression · Tetraploid · Triploid · Seedless · SRAP

\section{Introduction}

Siraitia grosvenorii (Swingle) C. Jeffrey, belonging to the genus Siraitia Merr under the family Cucurbitaceae, is a precious and economically important species endemic to southern China that and has been cultivated for several centuries. The fruits of $S$. grosvenorii, called Luohanguo, are used for food, beverages and traditional Chinese medicine. The fruit has evident effects on dry cough, sore throat, extreme thirst and constipation [1]. The major components of Luohanguo are mogrosides, a group of terpene glycosides, estimated to be about 300 times as sweet as sucrose [2]. One of the mogroside, mogroside V, is 400 times sweeter than sucrose [3]. Moreover, Luohanguo extracts have chemopreventive and antioxidant properties. Its non-caloric properties and effects on the cancer chemopreventive and antioxidant, being used as sweetening are being used as sweetening agents for the patients with diabetes mellitus, adiposis, hypertension, heart disease and so on. Luohanguo products have been approved as dietary supplements in Japan, the United States, New Zealand and Australia [4]. With the rapid rise in market demand, Luohanguo extracts have increased rapidly from two tons in 2002 to 60 tons in 2007, becoming one of the fastest growing traditional Chinese medicine extracts. 
Our group isolated a novel male mutant plant (M038) from hybrids (M036) of 'Bolin' and 'PinzhongND'. The mutant exhibited growth vigor, larger body and increased organ size compared to wild type. We then used pollen of M038 to pollinate 'Nongyuan'(a main cultivar), and the following year fruits of female hybrids (F050) were seedless (Fig. 1). Mogrosides only exist in flesh, occupying $15 \%$ of the dry weigh, whereas seeds constitute $70 \%$ of the weight but contain no mogrosides. Thus, seedless Luohanguo will no doubt increase the utilization and extraction rate of mogrosides. Therefore, mutant M038 and hybrid seedless Luohanguo are of great research value. M038 and F050 possesses distinct differences compared with wild type based on biological characters identified through our investigation: (1) M038 phenotypes include their strong growth potential. They are larger, have a thicker stem, wider and longer leaves, increased leaf area, bigger flowers and enhanced biomass. Both M038 and hybrid F050 have strong growth advantages as their flowering time, growing time and maturing time are all delayed. (2) The flower organ of M038 is particularly wider, while pollen is seriously aborted. Additionally, pollen quantity is less, while the germination rate of pollen is low. Most pollen is irregular and abnormal, shriveled and hollow. (3) Soluble sugar, glucose, mogroside, vitamin $\mathrm{C}$ and protein contents of F050 seedless fruits are all higher than in other main cultivars.

In this study, we carried out preliminary studies on karyotype analysis and the molecular mechanism of mutant M038. The results will provide reference information and resources for molecular breeding of seedless Luohanguo.
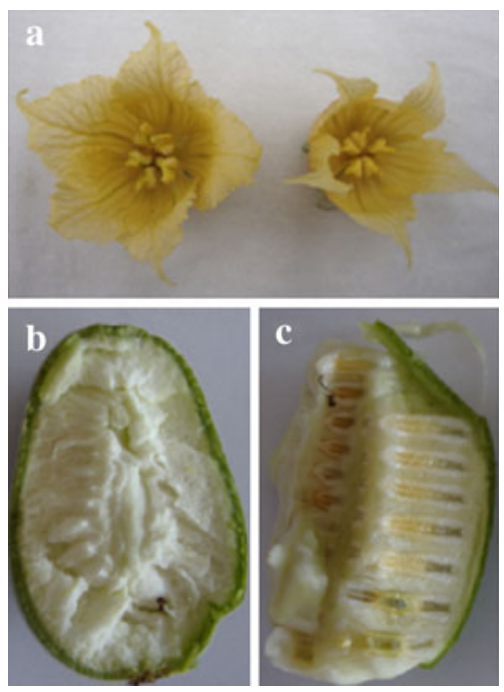

Fig. 1 a Flowers of $S$. grosvenorii mutant M038 and wild-type M036; b Fruits of seedless Luohanguo F050; c Fruits of normal Luohanguo

\section{Materials and methods}

Mutant M038, wild-type M036 and hybrid F050 were obtained from the Guangxi Branch Institute, Institute of Medicinal Plant Development, Chinese Academy of Medical Sciences \& Peking Union Medical College.

Karyotype analysis

Root tips $0.5-1 \mathrm{~cm}$ in length were collected from young plants and pretreated with saturated aqueous solution of p-dichlorobenzene at room temperature for $3 \mathrm{~h}$ before they were fixed in Carnoy (glacial acetic acid: absolute ethanol 1:3) for $1 \mathrm{~h}$. The tips were washed in distilled water, hydrolyzed in $1 \mathrm{mmol} / \mathrm{HCL}$ at $45^{\circ} \mathrm{C}$ for $45 \mathrm{~min}$, stained in phenol-fuchsin solution and squashed. The designation used to describe the karyotypes follows $\mathrm{Li}$ and Chen [5]. Karyotype symmetry was classified according to Stebbins [6].

\section{DNA extraction}

Genomic DNA was extracted from fresh leaves using the cetyltrimethylammonium bromide procedure [7]. Extracted DNA samples were dissolved in TE buffer and visualized after electrophoresis on $0.8 \% 1 \times$ TAE agarose gels. DNA purity and concentration was measured with a UV spectrophotometer and Nanodrop 8000. The DNA was adjusted to a final concentration of $30 \mathrm{ng} / \mu \mathrm{l}$ and stored at $-20^{\circ} \mathrm{C}$ until use.

RNA extraction and cDNA synthesis

Total RNA was isolated from pooled fresh leaves using the TRIzol reagent (Invitrogen, USA). All the RNAs were with treated by RNA-free DNase I and purified. RNA purity and concentration was measured with a UV spectrophotometer and Nanodrop 8000. M-MLV (RNase H) reverse transcriptase was used to synthesize cDNA following the manufacturer's protocol. Two technical replicates were performed.

SRAP analysis

A total of 196 different primer combinations were employed using 14 forward primers and 14 reverse primers (Supplementary Table 1). The primers sequences were synthesized according to $\mathrm{Li}$ and Quiros [8]. The PCR amplification for SRAP analysis were performed as follow: $94^{\circ} \mathrm{C}$ for $5 \mathrm{~min}$, followed by 5 cycles at $94^{\circ} \mathrm{C}$ for $45 \mathrm{~s}$, $35^{\circ} \mathrm{C}$ for $45 \mathrm{~s}$, and $72^{\circ} \mathrm{C}$ for $45 \mathrm{~s} ; 35$ cycles at $94^{\circ} \mathrm{C}$ for $45 \mathrm{~s}, 50^{\circ} \mathrm{C}$ for $45 \mathrm{~s}, 72^{\circ} \mathrm{C}$ for $45 \mathrm{~s}$ and a final extension at $72^{\circ} \mathrm{C}$ for $7 \mathrm{~min}$. The PCR products were analyzed using the 
QIAxcel capillary electrophoresis system, an automated analyzer offering high resolution and short analysis time. The system includes a device, cartridge, BioCalculator Analysis software and computer, and it applies capillary electrophoresis and fluorescence technology (Fig. 2).

Isolation and sequencing of differential fragments

The amplification products of the bands of interest were run on a $6 \%$ polyacrylamide gel at $45 \mathrm{~W}$ for $1.5 \mathrm{~h}$ in $1 \times$ TBE buffer and silver stained, and the bands were cut from the gel and eluted in $50 \mu \mathrm{l}$ of sterile double distilled water. About $5 \mu$ of the aliquot was reamplified using the same primer pairs and the same PCR conditions. The PCR products were ran in a $2 \%$ agarose gel and the bands were isolated and eluted using the gel extraction kit. Eluted fragments were cloned into plasmid pMD19-T propagated in E. coli DH5 cells and sent to Sangon biotechnology for sequencing.

\section{Sequences analysis}

The resulting sequences were compared to nucleotide and protein sequences in publicly available databases using BLAST sequence alignments. The functions of known genes identified by BLASTN and BLASTX searches were classified according to their putative functions.

\section{Results and discussion}

Karyotype analysis

The karyotypes of three materials studied are presented (Fig. 3). The chromosome numbers of M036, M038 and F050 were $2 n=2 x=28,2 n=4 x=56$ and $2 n=3 x=$ 42 , respectively. The results showed that wild-type M036 was a diploid strain, and the chromosome numbers $2 n=2 x=28$ were consistent with what has been previously been published on $S$. grosvenorii [9-11]. Mutant M038 was a tetraploid, and hybrid F050 was a triploid. Furthermore, M038 was an autopolyploid occurring within a species, relative to allopolyploidy following interspecific hybridization [6, 12]. To our knowledge, this is the first report on karyotype and molecular studied of polyploids in S. grosvenorii.

Genomic changes

Polyploidy, including autopolyploidy and allopolyploidy, may trigger changes in genome structure and gene expression [13]. At present, studies on polyploid plants have mainly concentrated on polyploid Arabidopsis, Brassica, Triticum, Gossypium, Nicotiana, Senecio, Spartina, Tragopogon and Triticale [14-28]. Most studies on polyploidization have focused on allopolyploid species
Fig. 2 QIAxcel capillary electrophoresis image of SRAP analysis. The top arrow indicates a band present in both the diploid and tertaploid strains; The middle arrow indicates a band present in the diploid strain but absent in the tetraploid one; The bottom arrow indicates a band present in the tetraploid strain but absent in the diploid; The D represents diploid M036; T represents tetraploid M038

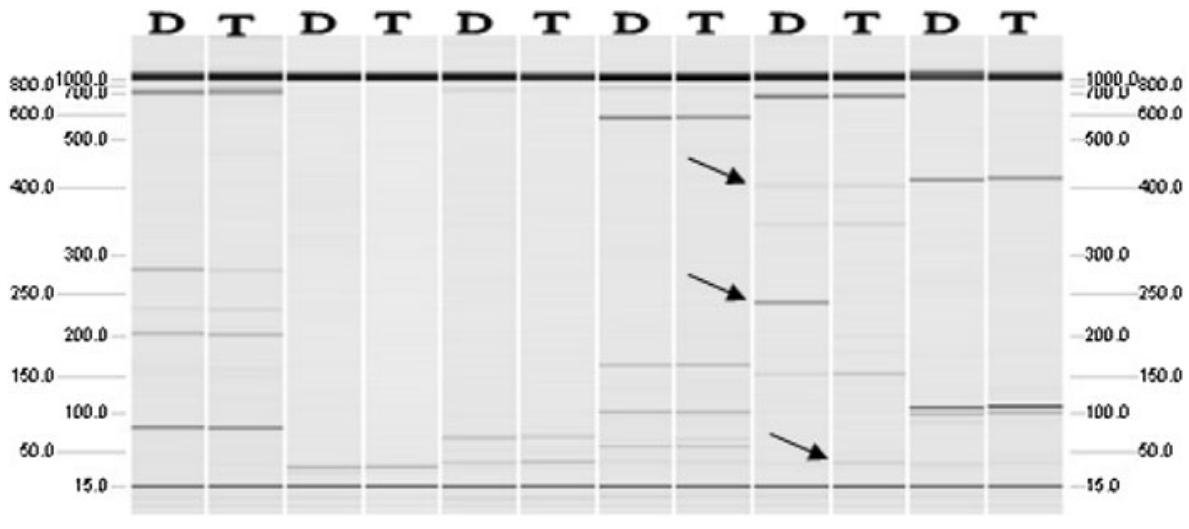

a

M038 and F050. a M036

$(2 n=2 x=28) ;$ b M038

$(2 n=4 x=56) ; \mathbf{c ~ F 0 5 0 ~}$

$(2 n=3 x=42)$
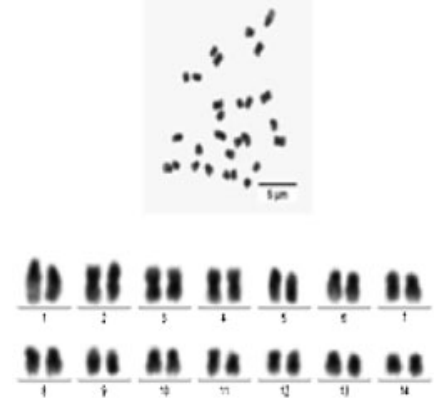

b

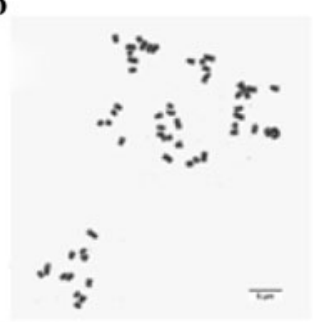

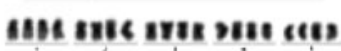

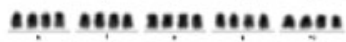

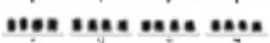

c

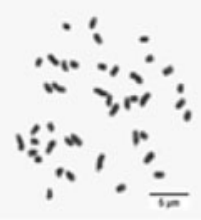

III III III 111 in

111 II " 11

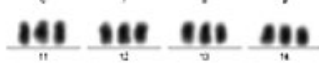


[17, 23, 28-31], whereas documents on autopolyploids are less [31-35]. Only a few studies dedicated to elucidating the consequences of autopolyploidization have been reported [34, 36-38].

A total of 196 SRAP primer combinations were used for DNA amplifications, nine combinations of which failed to amplify banding patterns. 189 SRAP primer combinations generated 4573 bands, $577(12.6 \%)$ of which were polymorphic while 1998 pairs $(87.4 \%)$ were identical. Fragment bands obtained were in the size range of 100-800 bp, while the number of bands amplified by each primer combination ranged from 6 (ME11a-OD3) to 19 (Me11-SA4), with a mean of 12.1. Moreover, The number of polymorphic bands for each primer combination varied from 0 (ME4-SA4) to 5 (Me9-Em18a), with a mean of 3.1. Based on the percentage of polymorphic bands, the levels of polymorphism ranged from 0 (Me12-Em10) to 37.6\% (PM8-Me10).

When chromosome doubling, genome structure and sequences have changed, it leads to differences and polymorphisms among diploid and tetraploid strains. According to conventional theory, It is expected that changes can not be observed during autopolyploidization. Our data also support general predictions. Only a small range of changes were observed in the genomes of tetraploid M038 compared to diploid M036. There were no apparent differences in the bands of M036 and M038 amplified by most primer pairs. The results generally indicated that the genetic diversity would be quite low between the diploid and tetraploid strains of $S$. grosvenorii.

\section{Expression changes}

Molecular results suggested that the rapid genome changes could accelerate evolutionary processes, and this may partly account for the appearance of many novel phenotypes. Further studies should focus on the characterization of the changed sequences and gene expression. Results of a wild autopolyploid sunflower series revealed that autopolyploidy does not appear to induce silencing or novel gene expression [39]. Addtionally, microarray analysis detected few changes associated with polyploidization [33]. The latest analysis of the transcriptome in an inbred maize ploidy series found frequent but low-level changes [40]. However, some articles demonstrated that autopolyploids also display differences in gene expression relative to diploids [17, 41].

A total of 196 SRAP primer combinations were used for cDNA amplifications, 63 of which failed to amplify any banding patterns. A total of 133 SRAP primer combinations generated 2917 bands, 289 (9.9\%) of which were polymorphic while 1313 pairs $(90.1 \%)$ were identical. Fragment bands obtained were in the size range of 100-800 bp. Stable and clearly differentiated fragments were sorted out, cloned and sequenced. Ninety-two differentially expressed fragments were successfully sequenced, $77.2 \%$ of them were highly homologous to known genes (Supplementary Table 2), 9.8\% were hypothetical genes and $13.0 \%$ were possible new genes as they had no significant similarity to known genes. The sequences encoding putative proteins were classified into 15 small groups based on previously reported gene functions and information from Gene Ontology (Fig. 4). These sequences provided useful and important molecular information for following studies on the expression changes between diploid and tetraploid of S. grosvenorii.

Natural autopolyploids and autopolyploids developed for agricultural purposes are often more vigorous and larger in size than their diploid relatives, indicating a fitness advantage and phenotypic superiority associated with higher ploidy [42]. Most studies on gene expression in polyploids have been based on phenotypic differences and related physiological characters, however, studies at the molecular level have not been widely carried out. Sequence analysis revealed that most fragments had significant homologous nucleotide sequence to photosynthesis, respiration and stress response genes. These genes included ribulose-1,5-bisphosphate carboxylase/oxygenase, phosphoenolpyruvate carboxykinase, pyruvate kinase, peroxisomal membrane transporter, NBS-LRR type resistance protein, protein phosphatase and others. The results, to some extent, indicated that the tetraploid of $S$. grosvenorii has more resistance to stress and photosynthesis ability than its diploid relatives. It provided molecular evidences for the phenomenon of
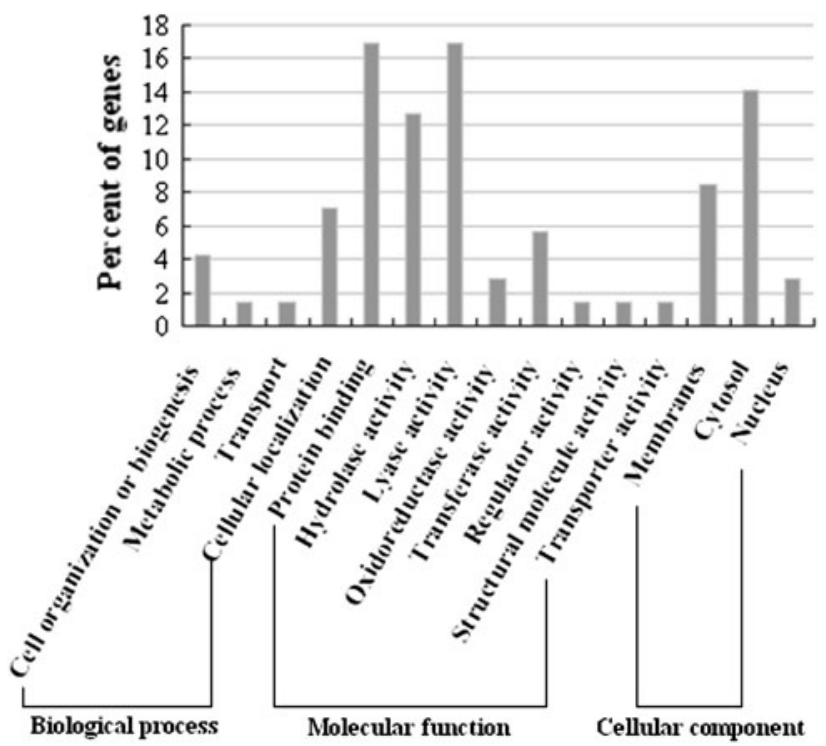

Fig. 4 Functional classification of differentially expressed fragments from cDNA-SRAP analysis based on gene functions and the GO annotation 
polyploids exceeding their diploid relatives based on the phenotypic and biological features.

Furthermore, functional analysis showed that the geneencoded proteins were involved in a broad range of biological pathways, including transporters, signal transduction, metabolism, transcription, protein synthesis, development, energy, etc. There proteins play important roles in regulating plant growth and development and include zinc finger protein, molecular chaperone, mitogen-activated kinase, transcription factor IWS1, transducin, endomembrane protein, porin, cellulose synthase, cytochrome P450, glycosyltransferase, oxidoreductase and other.

In summary, the interesting tetraploid mutant contains changes in genome structure and gene expression compared to the diploid strain. As this is the first study on tetraploid of S. grosvenorii., which will provide solid basis for future studies on tetraploid and hybrid triploid seedless Luohanguo. Future studies are planned to understand on more about genetic mechanism of triploid seedless Luohanguo.

Acknowledgments We are grateful to Professor Chen Jianbin from Nankai University for technical assistance in Karyotype analysis and the Key Laboratory of Tree Breeding and Cultivation, State Forestry Administration, Research Institute of Forestry for providing us with experimental support. This research was financially supported by the National Natural Science Foundation of China (Grant no. 30960500).

Open Access This article is distributed under the terms of the Creative Commons Attribution Noncommercial License which permits any noncommercial use, distribution, and reproduction in any medium, provided the original author(s) and source are credited.

\section{References}

1. Pharmacopoeia Commission of the People's Republic of China (2005) The pharmacopoeia of the People's Republic of China. Chemical industry press, Beijing

2. Kasai R, Nie RL, Nashi K, Ohtani K, Zhou J, Tao GD, Tanaka O (1989) Sweet cucurbitane glycosides from fruits of Siraitia siamensis (chi-zi luo-han-guo) a Chinese folk medicine. Agric Biol Chem 53(12):3347-3349

3. Matsumoto K, Kasai R, Ohtani K, Tanaka O (1990) Minor cucurbitane-glycosides from fruits of Siraitia grosvenorii (Cucurbitaceae). Chem Pharm Bull 38(7):2030-2032

4. Qin X, Xiaojian S, Ronggan L, Yuxian W, Zhunian Z, Shouji G, Heimbach J (2006) Subchronic 90-day oral (Gavage) toxicity study of a Luo Han Guo mogroside extract in dogs. Food Chem Toxicol 44:2106-2109

5. Li MX, Chen RY (1985) A Suggestion on the standardization of karyotype analysis in plants. J Wuhan Bot Res 3(4):297-305

6. Stebbins CL (1971) Chromosomal evolution in higher plants. Edward Arnold Ltd, London, pp 87-89

7. Sterward CN Jr, Via LEA (1993) Rapid CTAB DNA isolation technique useful for RAPD fingerprinting and other PCR application. Biotechniques 14:748-751

8. Li G, Quiros CF (2001) Sequence-related amplified polymorphism (SRAP), a new marker system based on a simple PCR reaction: its application to mapping and gene tagging in Brassica. Theor Appl Genet 103(2-3):455-461

9. Darlington CD, Wylie AP (1955) Chromosome atlas of flowering plants. George Allen \& Unwin Ltd, London

10. Zhuang WJ, Lin ZL, Zheng SK (1997) The chromosomes of Siraitia Grosvenorii. J Trop Subtrop Bot 5(2):23-25

11. Li Q, Ma L, Huang J, Li LJ (2007) Chromosomal localization of ribosomal DNA Sites and karyotype analysis in three species of Cucurbitaceae. J Wuhan Univ 53(4):449-456

12. Grant V (1971) Plant speciation. Columbia University Press, New York

13. Chen ZJ (2007) Genetic and epigenetic mechanisms for gene expression and phenotypic variation in plant polyploids. Annu Rev Plant Biol 58:377-406

14. Ozkan H, Levy AA, Feldman M (2001) Allopolyploidy-induced rapid genome evolution in the wheat (Aegilops-Triticum) group. Plant Cell 13:1735-1747

15. Shaked H, Kashkush K, Ozkan H, Feldman M, Levy AA (2001) Sequence elimination and cytosine methylation are rapid and reproducible responses of the genome to wide hybridization and allopolyploidy in wheat. Plant Cell 13:1749-1759

16. Adams KL, Cronn R, Percifield R, Wendel JF (2003) Genes duplicated by polyploidy show unequal contributions to the transcriptome and organ-specific reciprocal silencing. Proc Natl Acad Sci USA 100:4649-4654

17. Lee HS, Chen ZJ (2001) Protein coding genes are epigenetically regulated in Arabidopsis polyploids. Proc Natl Acad Sci USA 98:6753-6758

18. Kovarik A, Matyasek R, Lim KY, Skalicka K, Koukalova B, Knapp S, Chase M, Leitch AR (2004) Concerted evolution of 18-5.8-26S rDNA repeats in Nicotiana allotetraploids. Biol J Linn Soc 82:615-625

19. Pontes O, Neves N, Silva M, Lewis MS, Madlung A, Comai L, Viegas W, Pikaard CS (2004) Chromosomal locus rearrangements are a rapid response to formation of the allotetraploid Arabidopsis suecica genome. Proc Natl Acad Sci USA 101:18240-18245

20. Salmon A, Ainouche ML, Wendel JF (2005) Genetic and epigenetic consequences of recent hybridization and polyploidy in Spartina (Poaceae). Mol Ecol 14:1163-1175

21. Ma XF, Gustafson JP (2006) Timing and rate of genome variation in triticale following allopolyploidization. Genome 49:950-958

22. Tate JA, Ni ZF, Scheen AC, Koh J, Gilbert CA, Lefkowitz D, Chen ZJ, Soltis PS, Soltis DE (2006) Evolution and expression of homeologous loci in Tragopogon miscellus (Asteraceae), a recent and reciprocally formed allopolyploid. Genetics 173:1599-1611

23. Gaeta RT, Pires JC, Iniguez-Luy F, Leon E, Osborn TC (2007) Genomic changes in resynthesized Brassica napus and their effect on gene expression and phenotypic variation. Plant Cell 19:3403-3417

24. Kashkush K, Feldman M, Levy AA (2002) Gene loss, silencing, and activation in a newly synthesized wheat allotetraploidy. Genetics 160:1651-1659

25. Comai L, Tyagi AP, Winter K, Holmes-Davis R, Reynolds SH, Stevens Y, Byers B (2000) Phenotypic instability and rapid gene silencing in newly formed Arabidopsis allotetraploids. Plant Cell 12:1551-1567

26. Shang Yi, Ma L, Wang H, Feng W, Chen P, Cao X, Liu D, Wang $X$ (2011) The evolutionary history of PDR in Brachypodium distachyon polyploids. Mol Biol Rep 38:2211-2217

27. Luo L, Zhang J, Yang G, Li Y, Li K, He G (2008) Expression of puroindoline a enhances leaf rust resistance in transgenic tetraploid wheat. Mol Biol Rep 35:195-200

28. Zhuang Y, Chen JF, Jahn M (2009) Expression and sequence variation of the cucumber Por gene in the synthesized allotetraploid Cucumis $\times$ hytivus. Mol Biol Rep 36:1725-1731 
29. He P, Friebe BR, Gill BS, Zhou JM (2003) Allopolyploidy alters gene expression in the highly stable hexaploid wheat. Plant Mol Biol 52:401-414

30. Gaeta RT, Yoo SY, Pires JC, Doerge RW, Chen ZJ, Osborn TC (2009) Analysis of gene expression in resynthesized Brassica napus allopolyploids using arabidopsis 70mer oligo microarrays. PLoS One 4(3):e4760

31. Pires JC, Zhao J, Schranz ME, Leon EJ, Quijada PA, Lukens LN, Osborn TC (2004) Flowering time divergence and genomic rearrangements in resynthesized Brassica polyploids (Brassicaceae). Biol J Linn Soc 82:675-688

32. Xiao Y, Yu X, Chen J, Di P, Chen W, Zhang L (2010) IiSDD1, a gene responsive to autopolyploidy and environmental factors in Isatis indigotica. Mol Biol Rep 37:987-994

33. Pignatta D, Dilkes BP, Yoo SY, Henry IM, Madlung A, Doerge RW, Chen ZJ, Comai L (2010) Differential sensitivity of the Arabidopsis thaliana transcriptome and enhancers to the effects of genome doubling. New Phytol 186:194-206

34. Albertin W, Brabant P, Catrice O, Eber F, Jenczewski E, Chèvre AM, Thiellement H (2005) Autopolyploidy in cabbage (Brassica oleracea L.) does not alter significantly the proteomes of green tissues. Proteomics 5(8):2131-2139

35. Weiss H, Maluszynska $\mathbf{J}$ (2000) Chromosomal rearrangement in autotetraploid plants of Arabidopsis thaliana. Hereditas 133(3): 255-261
36. Guo M, Davis D, Birchler JA (1996) Dosage effects on gene expression in a maize ploidy series. Genetics 142:1349-1355

37. Galitski T, Saldanha AJ, Styles CA, Lander ES, Fink GR (1999) Ploidy regulation of gene expression. Science 285:251-254

38. Storchova Z, Breneman A, Cande J, Dunn J, Burbank K, O’Toole E, Pellman D (2006) Genome-wide genetic analysis of polyploidy in yeast. Nature 443:541-547

39. Church SA, Spaulding EJ (2009) Gene expression in a wild autopolyploid sunflower series. J Hered 100(4):491-495

40. Riddle NC, Kato A, Birchler JA (2006) Genetic variation for the response to ploidy change in Zea mays L. Theor Appl Genet 114:101-111

41. Lu B, Pan X, Zhang L, Huang B, Sun L, Li B, Zheng S, Yu X, Ding R, Chen W (2006) A genome-wide comparison of genes responsive to autopolyploidy in Isatis indigotica using Arabidopsis thaliana affymetrix chips. Plant Mol Biol Rep 24:197-204

42. Stupar RM, Bhaskar PB, Yandell BS, Rensink WA, Hart A, Shu O, Veilleux RE, Busse JS, Erhardt RJ, Buell CR, Jiang JM (2007) Phenotypic and transcriptomic changes associated with potato autopolyploidization. Genetics 176:2055-2067 